\title{
Blastocyst Culture and Embryo Transfer on Day 5 following Fertilization is a Viable Strategy for Acceptable Results in in vitro Fertilization: Intracytoplasmic Sperm Injection Program
}

\author{
${ }^{1}$ Rohan Palshetkar, ${ }^{2}$ Hrishikesh Pai, ${ }^{3}$ Biswanath G Dastidar, ${ }^{4}$ Nikita Lad, ${ }^{5}$ Jiteeka Thakkar, ${ }^{6}$ Nandita Palshetkar
}

\begin{abstract}
Despite considerable advances in the field of in vitro fertilization (IVF), embryo implantation and pregnancy rates have plateaued globally. Thus, much of current research focus is on embryo and endometrial assessment. Day 3 embryo transfers (ETs) have been the standard of practice for long. However, development of blastocyst culture media has led to recent switch toward blastocyst transfers, although this is associated with greater technical skill and know-how in order to optimize the culture process. We analyzed our blastocyst transfer results to see whether this strategy appeared to be a viable intervention in terms of acceptable pregnancy outcome. Our study found a pregnancy rate of $37.5 \%$ with blastocyst transfers, with the complete absence of any multiple pregnancies. Thus, we feel blastocyst transfers represent a viable intervention in an IVF program to ensure acceptable pregnancy rates and simultaneously reduce the incidence of multiple births.
\end{abstract}

Keywords: Blastocyst transfer, In vitro fertilization, Intracytoplasmic sperm injection.

How to cite this article: Palshetkar R, Pai H, Dastidar BG, Lad N, Thakkar J, Palshetkar N. Blastocyst Culture and Embryo Transfer on Day 5 following Fertilization is a Viable Strategy for Acceptable Results in in vitro Fertilization: Intracytoplasmic Sperm Injection Program. J South Asian Feder Obst Gynae 2018;10(2):131-133.

Source of support: Nil

Conflict of interest: None

Date of received: 15 March 2018

Date of acceptance: 20 April 2018

Date of publication: July 2018

\section{INTRODUCTION}

Despite tremendous advances in the field of IVF and assisted reproductive technology, embryo implantation

\footnotetext{
${ }^{1,4,5}$ Consultant, ${ }^{2,6}$ Director, ${ }^{3}$ Resident

${ }^{1,2,4-6}$ Department of Obstetrics and Gynecology, Bloom IVF Navi Mumbai, Maharashtra, India

${ }^{3}$ Department of Obstetrics and Gynecology, Dr D Y Patil Hospital \& Research Center, Navi Mumbai, Maharashtra, India

Corresponding Author: Rohan Palshetkar, Consultant Department of Obstetrics and Gynecology, Bloom IVF, Navi Mumbai, Maharashtra, India, e-mail: rohanpalshetkar88@ gmail.com
}

rates remain poor globally. ${ }^{1}$ The embryo and the endometrium are the two principal players involved in the process of embryo implantation. It has thus been a recent research focus to refine and develop more sophisticated techniques for embryo culture and assessment, as well as to assess endometrial receptivity.

Transfer of embryos at the cleavage stage on days 2 or 3 following fertilization has been the standard of practice for long. With the development of advanced blastocyst culture media and sequential media, it has become possible to culture embryos to the blastocyst stage. ${ }^{2}$

Survival of extended in vitro culture up to day 5 is in itself a marker of the developmental competence of embryos. ${ }^{3}$ Thus, embryos that survive to develop to the blastocyst stage are thought to be the best quality embryos. This process of "self-selection" implies that any embryos which grow to become blastocysts have greater odds to implant into the endometrium compared with day 3 embryos.

This also means that fewer blastocysts can be transferred into the uterus without compromising on the odds to achieve a pregnancy. Elective single ET has been shown to result in acceptable pregnancy rates, while simultaneously reducing the occurrence of multiple births, which are more common with the standard transfer of 2 to 3 or more day 3 embryos. ${ }^{4,5}$ Newer technology, such as preimplantation genetic screening also provides a potent tool to further assess these blastocysts to choose the best quality embryos for transfer. Thus, there has been recent opinion to shift to blastocyst transfers in IVF. However, blastocyst culture is technologically more challenging. Only a subset of day 3 embryos will go on to grow to blastocysts and in certain cycles, none will. ${ }^{6}$ This is also dependent on the technical expertise and quality control in individual laboratories. Thus, before switching from cleavage stage ETs to blastocyst transfers, an individual IVF laboratory needs to assess its level of technical skill, expertise, and know-how for the greater challenges involved in culturing an embryo up to blastocyst stage, and assess the risk of adversely affecting pregnancy rates in trying to switch to blastocyst transfers. 


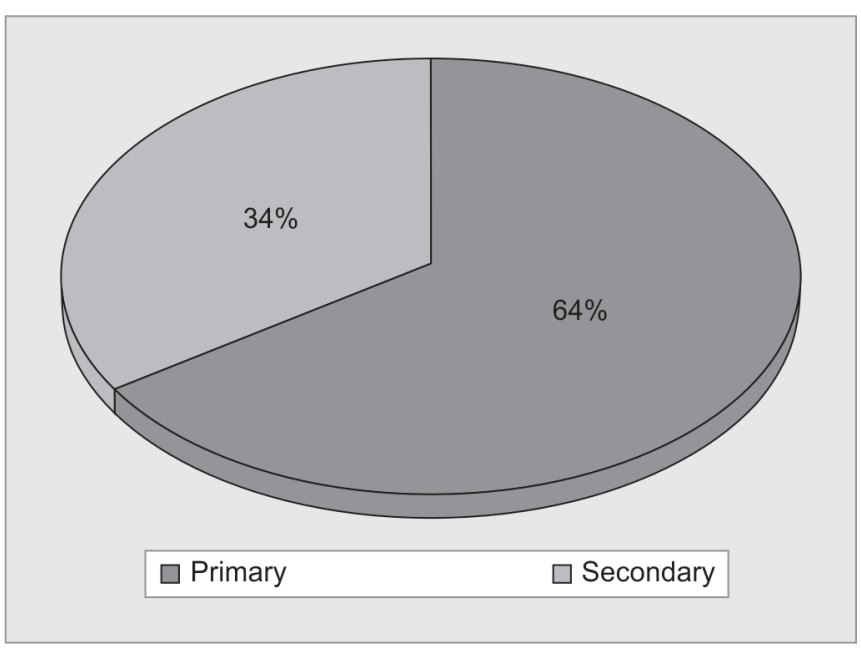

Graph 1: Incidence of primary and secondary infertility within the patient group

We retrospectively analyzed our results over 2 years of fresh blastocyst transfers in normo-responder, good prognosis patients below 37 years to assess whether it is a viable intervention strategy in terms of results.

\section{MATERIALS AND METHODS}

The study was done at an IVF center in Navi Mumbai between August 2015 and September 2017. Fresh day 5 blastocyst transfer data were analyzed. Institutional Ethics Committee approval was obtained. Informed consent was obtained from the patients. Patients below 37 years of age were included in the analysis. All patients were suffering from tubal or male factor infertility. Ovarian stimulation had been done with highly purified human menopausal gonadotropin (Menogon, Ferring Pharma, Switzerland) or recombinant follicle stimulating hormone (Gonal F, EMD Serono, USA). Stimulation monitoring was done by serial transvaginal ultrasound and serum $\mathrm{E}_{2}$ estimation. Daily gonadotropin-releasing hormone antagonist (Cetrotide $0.25 \mathrm{mg}$, EMD Serono, USA) started when lead follicle was $14 \mathrm{~mm}$ on ultrasonography (USG). Ovulation trigger given with recombinant human chorionic gonadotropin (hCG) (Ovitrelle $250 \mu \mathrm{g}$, Merck Serono, UK) once at least 2 to 3 follicles were $17 \mathrm{~mm}$ or more. Ovum pickup was done under USG guidance 36 hours after hCG. As per routine protocol, IVF or intracytoplasmic sperm injection had been done in all patients. As per standard protocol, USG-guided ET done on day 5 following fertilization, with 1 to 2 blastocysts were transferred per patient; 32 patients were part of the final data analysis. Luteal support was given with progesterone in oil injections on every 4th day (Gestone $100 \mathrm{mg}$, Nordic Pharma, UK), as well as vaginal micronized progesterone in the interim days (Crinone Gel, 8\%, Merck Serono, UK). Pregnancy was established by the documentation of fetal cardiac activity at 5 to 7 weeks of gestation.

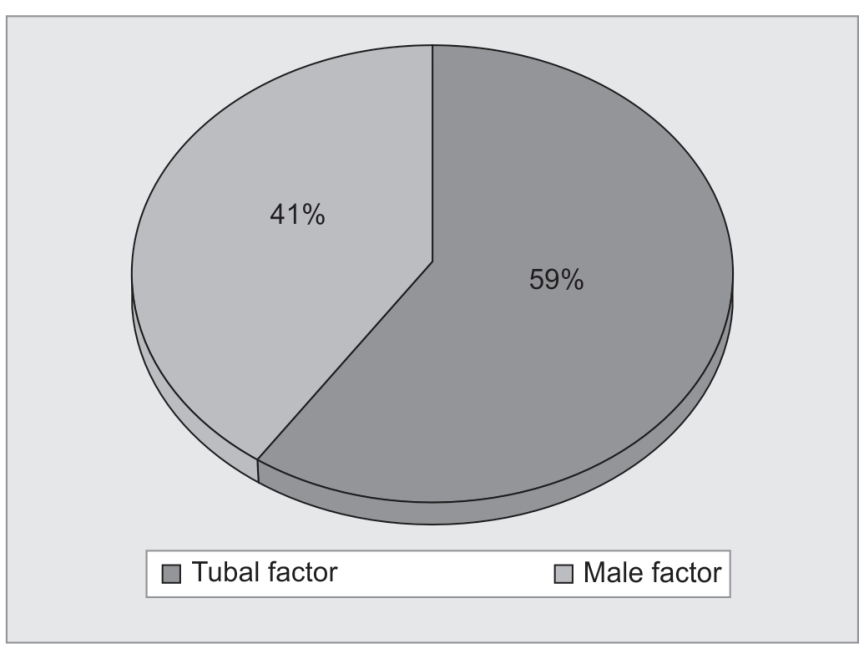

Graph 2: Cause of infertility within the patient group

Table 1: Summary of patient parameters and cycle results

\begin{tabular}{ll}
\hline Parameter & Result \\
\hline Age (years) & $30.7 \pm 3.47$ \\
AMH $(\mathrm{ng} / \mathrm{mL})$ & $2.61 \pm 0.96$ \\
Follicles aspirated & $8.91 \pm 3.51$ \\
Oocytes retrieved & $7.28 \pm 3.17$ \\
Embryos generated & $4.0 \pm 2.31$ \\
Fertilization rate & $54.3 \%$ \\
Endometrial thickness $(\mathrm{mm})$ & $9.97 \pm 1.81$ \\
No. of embryos transferred & $1.38 \pm 0.49$ \\
Implantation rate & $32.8 \%$ \\
Pregnancy rate & $37.5 \%$ \\
Multiple pregnancy rate & None \\
No. of embryos stored by vitrification & $1.25 \pm 1.7$ \\
\hline
\end{tabular}

\section{RESULTS}

Data analysis revealed that $66 \%$ of patients $(n=21)$ had presented with primary infertility and $34 \%$ with secondary infertility $(n=11)$ as seen in Graph $1 ; 59 \%$ patients had presented with tubal factor infertility, whereas $41 \%$ presented with male factor infertility, as seen in Graph 2. Other main results are summarized in Table 1.

Data analysis showed that mean age of patients was 30.7 years and mean anti-Mullerian hormone (AMH) level was $2.61 \mathrm{ng} / \mathrm{mL}$. A mean of 7.28 oocytes had been retrieved per patient. An average of 1.3 embryos had been transferred per patient. Mean endometrial thickness on day of ET was $9.97 \mathrm{~mm}$. We recorded a mean fertilization rate of $54.3 \%$, implantation rate of $32.8 \%$, and pregnancy rate of $37.5 \%$ in the cohort. There were no multiple pregnancies in the group. An average of 1.25 top quality embryos were available for freezing at the end of ET.

\section{DISCUSSION}

Our study had only included patients who had presented with infertility due to tubal or male factor. We had 
also designed the study to only include patients below the age of 37 years. Our data show that an acceptable pregnancy rate of $37.5 \%$ was achieved with blastocyst transfers on day 5 following fertilization. While it is true that pregnancy rates with blastocyst transfers can be even higher, these results demonstrate that for centers practicing day $3 \mathrm{ETs}$, it is possibly a viable alternative to switch to blastocyst transfers and achieve acceptable pregnancy rates.

The technical expertise required for blastocyst culture translates to a slightly steeper learning curve for the IVF embryologist. However, once the related skills are acquired, blastocyst transfers do appear to be a viable strategy for acceptable results in an IVF program. Moreover, none of the ETs resulted in a multiple pregnancy. We believe that it was possible to achieve this because of our conservative approach to ETs, where an average of only 1.3 blastocysts were transferred per patient.

Thus, in view of our acceptable pregnancy outcome and complete absence of multiple pregnancies, we feel that with adequate training in embryo culture techniques, blastocyst culture and ET on day 5 following IVF is a viable strategy to ensure good results and, at the same time, mitigate the risk of multiple births. However, our sample size was small. Thus, larger, well-designed trials are required to provide more evidence in support of our findings and conclusions.

\section{REFERENCES}

1. Calhaz-Jorge C, De Geyter C, Kupka MS, de Mouzon J, Erb K, Mocanu E, Motrenko T, Scaravelli G, Wyns C, Goossens V. Assisted reproductive technology in Europe, 2013: results generated from European registers by ESHRE. Hum Reprod 2017 Oct 1;32(10):1957-1973.

2. Germond M, Wirthner D, Senn A, Lausanne Consensus Meeting on National ART Registers. Core data for assisted reproductive technology registers: results of a consensus meeting. Reprod Biomed Online 2008 Dec;17(6):834-840.

3. George K, Mangalraj A, Muthukumar K, Aleyamma T, Kamath M. Blastocyst stage transfer vs cleavage stage embryo transfer. J Hum Reprod Sci 2009;2(1):23.

4. Criniti A, Thyer A, Chow G, Lin P, Klein N, Soules M. Elective single blastocyst transfer reduces twin rates without compromising pregnancy rates. Fertil Steril 2005;84(6):1613-1619.

5. Gelbaya TA, Tsoumpou I, Nardo LG. The likelihood of live birth and multiple birth after single versus double embryo transfer at the cleavage stage: a systematic review and metaanalysis. Fertil Steril 2010;94(3):936-945.

6. Dessolle L, Fréour T, Barrire P, Daraï E, Ravel C, Jean M, Coutant C. A cycle-based model to predict blastocyst transfer cancellation. Hum Reprod 2010;25(3):598-604. 